Artículo original

\title{
CARACTERIZACIÓN DE RESIDUOS SÓLIDOS EN EL CAMPUS CAPANIQUE DE LA UNIVERSIDAD PRIVADA DE TACNA
}

CHARACTERIZATION OF SOLID WASTE IN THE CAPANIQUE CAMPUS OF THE PRIVATE UNIVERSITY OF TACNA

Richard Sabino Lazo Ramos ${ }^{1}$

Milagros Herrera Rejas ${ }^{2}$

Información del artículo:

Recibido: 18/08/2019

Aceptado: 05/02/2020

${ }^{1,2}$ Escuela Profesional de Ingeniería Ambiental. Facultad de Ingeniería, Universidad Privada de Tacna, Perú. E-mail: ${ }^{1}$ ozalsomar@gmail.com, ${ }^{2}$ milaherrerar@gmail.com 


\section{Resumen}

El estudio tuvo como propósito estudiar las características y conocer la cantidad de los residuos sólidos generados por la comunidad universitaria en el campus Capanique de la Universidad Privada de Tacna (UPT). Se aplicó la normativa para residuos sólidos municipales del MINAM (2012). La obtención de la muestra fue aplicando el método del cuarteo el cual permitió conocer las características del material reciclable y orgánico que fue de un $59,0 \%$ del total de residuos sólidos, dentro de ellos el PET (botellas de plástico) con el $14,52 \%$ y la materia orgánica con el $10,93 \%$. Referente a la cantidad de residuos generados se determinó el peso volumétrico o densidad "in situ" que fue de $102,3 \mathrm{~kg} / \mathrm{m}^{3}$ lo que señala una baja densidad de residuos en los depósitos de la Universidad, así mismo se estimó la tasa de generación per cápita que fue de 0,5 ( $\mathrm{kg} / \mathrm{d}$ dí/persona). Según el grupo de generación de residuos se demostró que la mayor cantidad de residuos fueron generados en el área administrativa y por los docentes a tiempo completo 0,11 ( $\mathrm{kg} /$ día/persona), además el campus que generó mayor residuo fue el campus 3 constituido por la Facultad de Ciencias Empresariales con 190,932 kg/semana y la generación de los residuos hospitalarios procedente de la Clínica Universitaria de la Facultad de Ciencias de la Salud fue de 1235 $\mathrm{kg} / \mathrm{día}$. La investigación significa una contribución para una adecuada gestión de residuos sólidos en la Universidad Privada de Tacna.

Palabras claves: características de residuos sólidos; generación per cápita; peso volumétrico.

\section{Abstract}

The purpose of the study was to study the characteristics and know the amount of solid waste generated by the university community in the Capanique campus of the Private University of Tacna (UPT). For this study, the municipal solid waste regulations of the MINAM (2012) were applied. The obtaining of the sample was applying the method of the cuarteo which allowed to know the characteristics of the recyclable and organic material that was of a $59,0 \%$ of the total of solid residues, inside them the PET (plastic bottles) with the $14,52 \%$ and organic matter with $10,93 \%$. Regarding the amount of waste generated the volumetric weight or density "in situ" was determined, which was $102.3 \mathrm{~kg} / \mathrm{m}^{3}$, which indicates a low density of residues in the deposits of the University. Likewise, the generation rate was estimated. per capita that was 0.5 ( $\mathrm{kg} /$ day / person). According to the waste generation group it was shown that the largest amount of waste was generated in the administrative area and by full-time teachers 0.11 ( $\mathrm{kg} /$ day / person), in addition the campus that generated the most waste was the campus 3 constituted by the Faculty of Business Sciences with 190,932 kg / week and the generation of hospital waste from the University Clinic of the Faculty of Health Sciences was $1235 \mathrm{~kg} /$ day. The present investigation means a contribution for an adequate management of solid waste in the Private University of Tacna

Keywords: characteristics of solid waste; generation per capita; volumetric weight. 


\section{Introducción}

La producción de residuos sólidos es común en lugares con presencia de población y su cantidad cambia de acuerdo componentes culturales, relacionados a la situación económica, prácticas de consumo, avance tecnológico y a la calidad de vida. El campus Capanique de la Universidad Privada de Tacna posee con una población de aproximadamente 5057 personas, que permanecen 8 horas al día como promedio en salones de clase, áreas administrativas, laboratorios, bibliotecas, cafeterías, etc., los cuales son usados por los estudiantes, docentes, personal administrativo, de limpieza, de seguridad, entre otros, generando por ende, residuos sólidos.

La caracterización de los residuos sólidos permiten determinar el tipo de residuos y la cuantificación estimará la cantidad de residuos sólidos que se generan diariamente, estos datos facilitan a reutilizar al máximo los residuos sólidos y realizar la disposición de una manera segura. Actualmente, se carece de una caracterización de los residuos sólidos generados en la Universidad Privada de Tacna, lo cual es un impedimento de realizar futuras tomar acciones que permitan la adecuada minimización, segregación y la disposición en el relleno sanitario y/o la incineración.

\section{Objetivos}

Realizar un diagnóstico de la generación residuos sólidos a partir de las actividades de la Universidad, el cual permitirá conocer sus características y generación per cápita en este centro de estudios y de ésta manera, según los resultados, las autoridades de la Universidad podrán tomar acciones en la definición de una política de una adecuada gestión ambiental para el manejo de residuos de manera sostenible e integral generados en las diferentes instalaciones que se encuentran en el campus universitario, con la finalidad de obtener mejoras en el componente socioambiental, y en consecuencia, en el estilo de vida de la comunidad universitaria.

\section{Metodología}

El diseño se basó en la aplicación de la normativa para residuos sólidos municipales del MINAM (2012). Se estudió la generación de residuos sólidos de una población de 5699 personas, el cual estuvo conformado por personal administrativo, estudiantes, docentes, entre otros, distribuidos según la tabla 1.

El estudio de caracterización se realizó durante 7 días consecutivos, desde el 3 al 9 de agosto del 2015 y según la metodología no se consideró la muestra obtenida el primer día, por la razón de desconocer la cantidad de residuos sólidos almacenados en los días precedentes.

Para una mejor aplicación de la metodología se delimitó espacialmente la Universidad Privada de Tacna en 3 sectores, el campus 1 constituido por la Facultad de Ingeniería (FAING), la Facultad de Arquitectura y Urbanismo (FAU) y la Facultad de Educación Ciencias de la Comunicación y Humanidades (FAEDCOH); el campus 2: Facultad de Derecho y Ciencias Políticas (FADE) y la Facultad de Ciencias de la Salud (FACSA) y el campus 3 por la Facultad de Ciencias Empresariales (FACEM). 
Tabla 1

Distribución de la población de la Universidad Privada de Tacna

\begin{tabular}{lccc}
\hline Campus & Estudiantes & Administrativos & $\begin{array}{l}\text { Docentes a } \\
\text { tiempo } \\
\text { completo }\end{array}$ \\
\hline $\begin{array}{l}\text { Capanique I FEDCOH, } \\
\text { FAING, }\end{array}$ & 2271 & 32 & 70 \\
FAU & & 12 & 10 \\
Capanique II(*): FADE & 864 & 18 & 30 \\
Capanique III: FACEM & 1750 & 62 & 110 \\
Subtotal & 4885 & Total & 5057 \\
\hline
\end{tabular}

Nota: Datos obtenidos del centro de Computo., Área de Personal-UPT, 2015.

Se entregó al personal de limpieza bolsas plásticas, además, se identificó 6 zonas de generación de residuos sólidos como: Oficinas administrativas, laboratorios, salones de clase, bibliotecas, cafetería y servicios higiénicos.

Se distribuyeron bolsas de 75 litros para el personal de limpieza para cada sector identificado a fin de que viertan los residuos sólidos generados en el campus. Cada bolsa fue marcada con un rotulador permanente la cual se indicó la fecha y zona de generación de los residuos sólidos. Dichas bolsas se colectaron por cada día y se sustituyeron por otras bolsas con colores característicos de acuerdo al lugar de procedencia con la finalidad de determinar cuál es el lugar donde se generan mayor cantidad de residuos sólidos.

\section{Tabla 2}

Clasificación de colores según la zona de generación de residuos sólidos

\begin{tabular}{lc}
\hline \multicolumn{1}{c}{ Color de bolsa } & Tipo de RR.SS \\
\hline Negra & Aulas (salones de clase, laboratorios, \\
& bibliotecas) \\
Amarilla & Servicios higiénicos \\
Verde & Cafetería \\
Celeste & Oficinas administrativas \\
\hline
\end{tabular}

Nota. RR. SS: residuos Sólidos. Elaboración propia, 2015

Cada día los residuos recolectados fueron trasladados a una zona asignada por el responsable del estudio. En dicho lugar, se procedió a la caracterización y cuantificación de los residuos.

Con los datos obtenidos, se estableció la cantidad de residuos sólidos per-cápita. Se aplicó la ecuación 1 ( MINAM, 2012) en donde se registró el peso total de residuos sólidos por día el cual dividió por la cantidad de personas de la Universidad Privada de Tacna, el resultado es la generación per-cápita por día de residuos sólidos obtenidos en 6 días. Los resultados se expresaron en kilogramos por persona por día (Kg/persona/día). 


$$
\text { GP }_{\text {ci }_{i}=\frac{\text { Díal+Día2+Día3+Día4+Día5+Día6 }}{\text { Número de alumnos } \times 6 \text { días }}}
$$

Con los resultados conseguidos a partir de la ecuación 1, se relacionaron con la cantidad de residuos sólidos generados por cada actividad universitaria (educativa, administrativa entre otras) empleando la ecuación 2.

Finalmente, cada subproducto se pesó por separado. Se aplicó la ecuación (3) según la normatividad, referente al cálculo del porcentaje en peso para cada subproducto.

$$
S=\frac{W}{v}=\frac{W}{\pi\left(\frac{D}{2}\right)^{2}(H-h)}
$$

Donde:

W: peso de los residuos solidos

$\mathrm{V}$ : volumen del residuo solido

D: diámetro del cilindro

$\mathrm{H}$ : altura del cilindro

$\mathrm{H}$ : Altura libre de residuos solidos

П: constante $(3,1416)$

Para la caracterización se mezclaron uniformemente todos los residuos, luego del pesaje diario, sobre una superficie cubierta por un plástico. Se dividió los residuos por cuarteo en 4 partes, se seleccionaron 2 partes opuestas y las otras dos partes se rechazaron.

Así mismo respecto a densidad de los residuos sólidos y peso volumétrico, con la primera parte producto del cuarteo, se efectuó la prueba de peso volumétrico. En la cual se recogieron los residuos para luego verterlos sin comprimirlos en cilindros de 220 litros los cuales fueron medidos y pesados. Se levantó unos $10 \mathrm{~cm}$. al nivel del suelo para luego dejarlo caer tres veces consecutivas, para homogeneizar la muestra. Luego se midió el volumen de los residuos y su peso total, descontándose del peso inicial del recipiente sin residuos sólidos. Los resultados se expresaron en kilogramos por metro cúbico $(\mathrm{kg} / \mathrm{m} 3)$, la cual se registró en formatos. La densidad se calculó, utilizando la fórmula 4 propuestas por MINAM 2013.

\section{$P V=\underline{\text { Díal+Día2+Día3+ Día4+Día5+Día6 }}$ \\ 6}

Con la segunda parte seleccionada, se realizó la prueba de caracterización que corresponde a la composición porcentual física de acuerdo al tipo de residuos sólidos, la cual consistió en seleccionar los residuos sólidos de acuerdo a su naturaleza física como: metal, vidrio, papel, plástico, entre otros; los cuales fueron pesados por separado.

Los tipos de residuos sólidos se registraron de acuerdo al peso por cada elemento, pudiéndose estimar su proporción del total, los datos obtenidos se expresaron en porcentaje. 


\section{Resultados}

Los residuos sólidos generados diariamente en el campus Capanique de la Universidad Privada de Tacna fue de $81,69 \mathrm{~kg} /$ día (0,081 toneladas) y la producción per cápita promedio fue de $0.05 \mathrm{Kg} / \mathrm{hab} /$ día.

\section{Tabla 4}

Generación de Residuos Sólidos del Campus Capanique de la UPT

\begin{tabular}{lc}
\hline \multicolumn{1}{c}{ Generación de Residuos Sólidos } & Cantidad \\
\hline Población universitaria(Hab)* & 5057 \\
Ppc (Kg/Hab/día) & 0,05 \\
kilogramo/día & 81,69 \\
Toneladas/día & 0,08169 \\
Tonelada/mes & 24,705 \\
Tonelada/año & 294,084 \\
\hline
\end{tabular}

Nota: $\left({ }^{*}\right)$ La comunidad universitaria corresponde los alumnos, docentes, administrativos, comensales, entre otros Ppc: producción per cápita. Elaboración propia, 2015.

Un personal de la Universidad Privada de Tacna (UPT) puede producir 0,05 kg/hab/día de residuos. En el día podría no parecer un aporte significativo, sin embargo, al calcularlo en meses y años, la proporción va en aumento. Al mes se tiene 2,4507 toneladas y al año llega a las 29,408 toneladas. Esta es la cantidad aproximada de residuos generados por la UPT.

Respecto a la generación per cápita total por áreas de trabajo en el campus Capanique de la Universidad Privada de Tacna, los datos se agruparon de acuerdo a las áreas y se utilizó la fórmula de la ecuación (2) estimándose la Generación per cápita de residuos sólidos total del campus Capanique de la Universidad Privada de Tacna

\section{Tabla 5}

Generación per cápita total por áreas del campus Capanique de la Universidad Privada de Tacna.

\begin{tabular}{lc}
\hline \multicolumn{1}{c}{$\begin{array}{c}\text { Generación per cápita de } \\
\text { residuos sólidos }\end{array}$} & $\begin{array}{c}\text { Cantidad } \\
\text { (Kg/día/persona) }\end{array}$ \\
\hline Alumnos & 0,011 \\
Profesores y administrativos & 0,112 \\
Comensales & 0,028 \\
Campus universitario & 0,050
\end{tabular}

Nota. Elaboración propia, 2015. 
Los resultados obtenidos muestran que la mayor producción per cápita por áreas de trabajo fue la correspondiente al área administrativa, docentes con 0,112 kg/día/persona.

\section{Tabla 6}

Generación de residuos sólidos por sectores

\begin{tabular}{lc}
\hline Generación de residuos sólidos & $\begin{array}{c}\text { Cantidad } \\
(\mathrm{kg})\end{array}$ \\
\hline $\begin{array}{l}\text { Campus1 } \\
\text { (FAING-FAU_FAEDCOH) }\end{array}$ & 189,643 \\
Campus 2 & 190,566 \\
(FADE-FACSA) & \\
Campus 3 & 190,932 \\
(FACEM) & \\
Total UPT Campus Capanique & 490,141 \\
Peso diario Campus Capanique & 81,69 \\
\hline
\end{tabular}

Nota. Elaboración propia, 2015.

El campus de la UPT genera $81,69 \mathrm{~kg} /$ día que genera más residuos en la Universidad es el Campus 3 Facultad de Ciencias Empresariales con 190,932 kg/semana, el cual, es el segundo con mayor concentración poblacional.

La Densidad de los residuos sólidos y el peso volumétrico, se obtuvo al medir la altura libre de los residuos sólidos en un cilindro dimensionamiento conocido resultando $102,3 \mathrm{Kg} / \mathrm{m}^{3}$, lo que señala un bajo volumen de los desechos sólidos dispuestos en los depósitos o tachos de basura, probablemente debido al contenido más livianos de envases de alimentos y bebidas que se consume en las cafeterías de la Universidad, a la vez, que no hay una alta cantidad de materia orgánica u otros desechos de alto peso como vidrio, etc. Pero es necesario realizar este mismo estudio en otras épocas en la que existen otras actividades tales como eventos, festivales o campeonatos en las que pueden hacer variar los datos.

El empleo de este valor es necesario para plantear un programa de implementación contenedores para residuos sólidos en el campus de la universidad.

Los valores obtenidos durante la semana de evaluación en cuanto al tipo de residuos producto de la segregación, siendo los resultados de mayor porcentaje de generación fueron los residuos inorgánicos con el 89,07 \% y los orgánicos con el 10,93\%.

Del porcentaje total de residuos inorgánicos, el papel fue el 19,17\%, seguido de residuos sanitarios o llámese papel de baño representó el $18,73 \%$, siendo el segundo en mayor cantidad, lo que debe tomarse en cuenta por no representar beneficio económico, lo cual, debe manejarse en forma distinta ya que puede representar un riesgo y su manejo se debe considerar como residuo peligroso. 


\section{Figura 1}

Composición de los residuos sólidos del Campus Capanique UPT

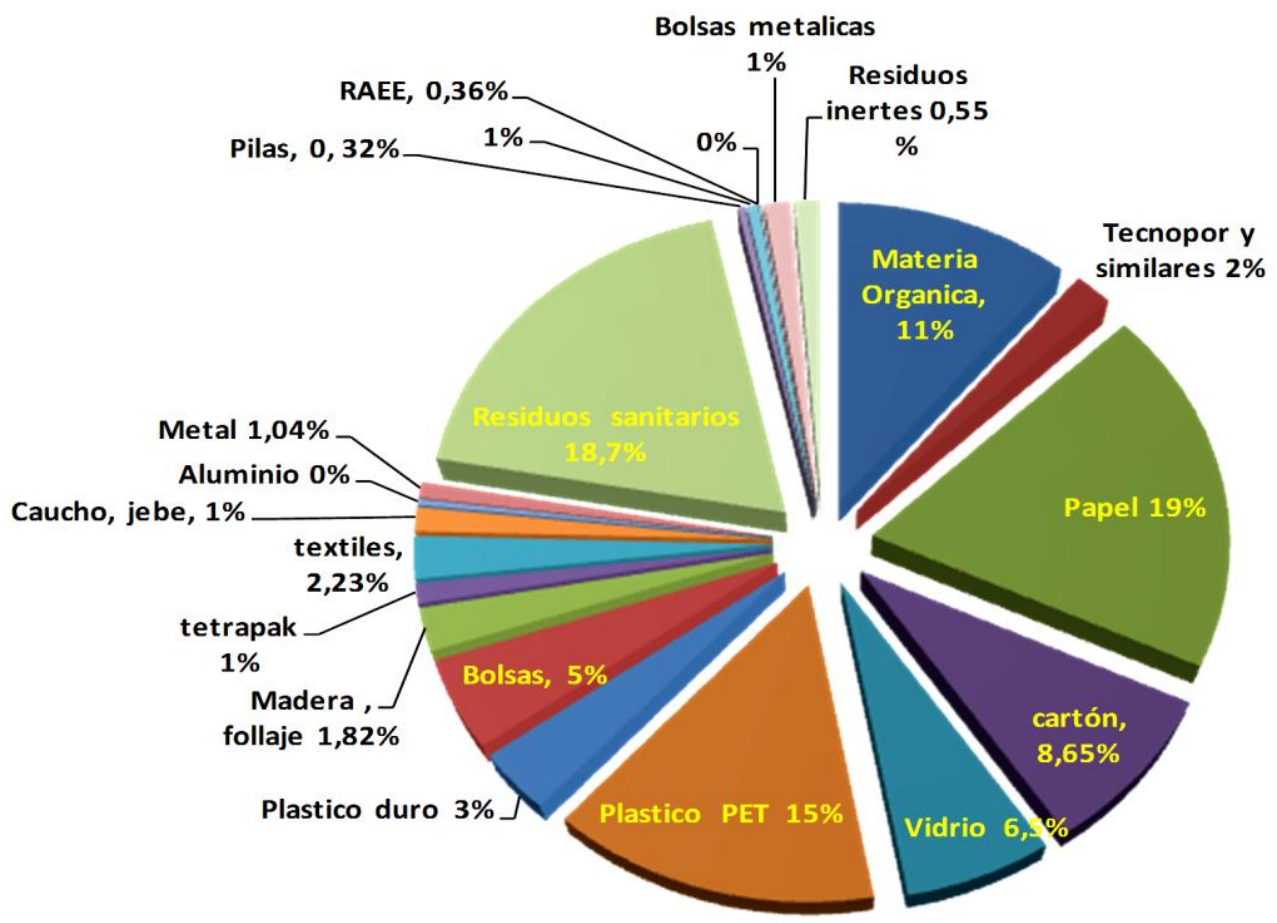

Nota. Elaboración propia, 2015.

También existen otros residuos clasificados como material reciclable que están en mayor cantidad (plástico PET, materia orgánica, cartón, vidrio, bolsas de plástico, plástico duro, tecnopor) que representa un $51,18 \%$, siendo el más predominante; el plástico PET con 14,52 \%, materia orgánica con un $10,93 \%$, Cartón con $8,65 \%$, Vidrio con $6,55 \%$, seguido de Bolsas de plástico con un $4,86 \%$, Plástico duro 3,39\%, tecnopor y similares $2,28 \%$,

Asimismo, el estudio muestra los siguientes materiales los cuales representaban un porcentaje muy bajo individualmente por lo que se decidió agruparlos aunque no representan un beneficio económico en caso de reciclado (Telas textiles $2,23 \%$, madera, follaje $1,82 \%$, caucho y cuero $1,26 \%$, tetrapack $1,21 \%$, bolsas metálicas $1,22 \%$, aluminio $0,10 \%$ y otros residuos en mucho menores proporciones).

Estos indicadores muestran los hábitos de consumo y generación de residuos por parte de los alumnos, profesores y administrativos, es muy destacable apreciar que más del $50 \%$ de los residuos de la UPT corresponde a material reciclable y orgánico.

\section{Discusión}

La generación per cápita del campus Capanique de la Universidad Privada de Tacna fue de $0,05 \mathrm{~kg} /$ pers/día, la cual, está muy por debajo de otras universidades como la Universidad Mayor de San Marcos 0,2 kg/persona/día (Canchari \& Ortiz, 2007) o extranjeras como la Universidad Iberoamericana con una población aproximada de 10000 personas con una generación per cápita de 0,33kg (Morales, 2011), y en la Universidad Autónoma de México fue de 0,11 kg/persona (Alcántara et al.,2003) 
Sin embargo, cifras similares de la generación de residuos de nuestra universidad se encontraron en el estudio de la Facultad de Ciencias Forestales de la Universidad de Los Andes en Venezuela 0,075 kg/hab./día (Dávila \& Unshelm, 2011) y en la Universidad Autónoma de Baja California (México) con $0,05 \mathrm{Kg} /$ persona y $0,003 \mathrm{Kg} / \mathrm{m}^{2}$ (Arnijo, 2006)

El índice de Generación Per Cápita total ( $0.05 \mathrm{Kg} / \mathrm{Hab}$./día), está en el rango de 0.050 a 0.250 (Kg/Día/Persona) en instituciones universitarias, lo que muestra que existe una cultura muy aceptable en cuanto a generación de residuos sólidos (Dávila \& Unshelm, 2011).

Los resultados obtenidos difieren a la generación de residuos domiciliarios, cuyo rango determinado por MINAM (2008), es de 0,5 a 0,7 Kg/hab/día, esto debido a que la cantidad de los residuos orgánicos se presentan mucho más en las viviendas y esto hace que aumente el peso de los residuos. Es importante contar con esta información a fin de establecer si existen diferencias en la generación de residuos sólidos en estudiantes de universidades privadas y públicas.

El campus de la Universidad Privada de Tacna generó 81,69 kg/día. Sin embargo, en la ciudad universitaria de Michoacana en México estudiaron que el peso de los residuos de las 499 personas muestreadas fue de 155,75 Kg/día (Carrillo, 2007), la cual es mucho mayor que la nuestra, demostrando así que la cultura en nuestra universidad en mejor ya que la población universitaria del campus Capanique de la UPT supera al doble a la Universidad de Michoacana. Pero resultado similar se encontró en la Facultad de Ciencias Forestales de la Universidad de Los Andes la cantidad de residuos por día es de $83.60 \mathrm{~kg}$ (Runfola \& Gallardo, 2014).

Dentro de los residuos inorgánicos de la Universidad Privada de Tacna, el más relevante fue el papel con un 19,17 \%, el cual es bajo en comparación con otras universidades como la Universidad Nacional Mayor de San Marcos con 60\% (Canchari, \& Ortiz, 2007) y la Universidad Michoacana de San Nicolás de Hidalgo con 38,28\% de residuos de papel, (Carrillo, 2007), estos residuos se generan regularmente en ciudad universitaria como producto de sus actividades académicas, administrativas, operacionales o de prestación de servicios, siendo un material fácilmente reciclable.

\section{Conclusiones}

La producción per cápita en el Campus Capanique fue de 0,050 Kg/Hab/día. Se proyecta la producción total - día de residuos sólidos de $81,69 \mathrm{~kg} /$ día (0,081.69 Toneladas/día) para del campus de la Universidad Privada de Tacna, siendo el peso volumétrico o densidad de los residuos sólidos universitarios fue de $102,3 \mathrm{Kg} / \mathrm{m}^{3}$.

La composición física demostró que el residuo de mayor generación en el campus Capanique es el papel con un $19,17 \%$, seguido de residuos sanitarios con $18,73 \%$, plástico PET $14,52 \%$, materia orgánica con un $10,93 \%$, cartón con $8,65 \%$, vidrio en 6,55 , seguido de bolsas de plástico con un $4,86 \%$, plástico duro $3,39 \%$, tecnopor y similares $2,28 \%$, telas textiles $2,23 \%$, madera follaje $1,82 \%$, caucho y cuero $1,26 \%$, tetrapack $1,21 \%$, bolsas metálicas $1,22 \%$, aluminio $0,10 \%$ y otros residuos en mucho menores proporciones.

Los residuos sólidos con alto potencial de reciclaje determinados en el estudio son el papel, plástico PET, materia orgánica, cartón, vidrio representando un total de $59,0 \%$ de los residuos sólidos del campus de la UPT. 


\section{Referencias bibliográficas}

Alcántara Concepción, Víctor; Cruz Gavilán García, Irma; Santos, Elvira (2003). Caracterización y cuantificación de los residuos sólidos en ciudad universitaria. Unidad de Gestión Ambiental, Facultad de Química, UNAM, Ciudad Universitaria, Coyoacán, México DF.

Armijo, C. (2006). Potencial de reciclaje de los residuos de una institución de educación superior; el caso de la Universidad de Baja California Ingeniería.

Carrillo Mainé, Núria. (2007). Análisis de los residuos sólidos generados en áreas administrativas, académicas, bibliotecas y cómputos, de ciudad universitaria en la Universidad Michoacana de San Nicolás de Hidalgo (UMSNH), Michoacán, México.

Canchari Silveri, Godelia; Ortiz Sánchez, Oswaldo. (2007). Valorización de los residuos sólidos en la ciudad universitaria de la Universidad Nacional Mayor de San Marcos Revista del Instituto de Investigaciones FIGMMG Vol. 11, № 21, (2008) UNMSM.

Dávila Rangel, Arsenio; Unshelm Báez Carlos. (2011). Monitoreo y Caracterización de los Residuos y Desechos Sólidos de Índole no peligrosos generados en la Facultad de Ciencias Forestales y Ambientales En Mérida. Venezuela. Misredes artículo científico.

MINAM. (2012). Guía metodología para la elaborar e implementar un programa de segregación selectiva de residuos sólidos municipales. Lima.

MINAM. (2008). Informe de la Situación Actual de la Gestión de Residuos Sólidos Municipales. [En línea]: (http:// www.minam.gob.pe, 30 Ag. 2011)

Ruiz Morales, Mariana. (2011). Caracterización de residuos sólidos en la Universidad Iberoamericana, Ciudad de México. [Documento en línea]. Portal de revistas

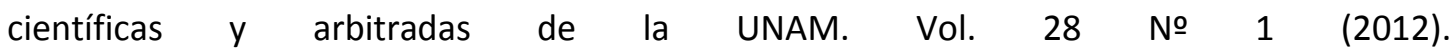
http://www.recercat.cat/bitstream/handle/2072/5324/PFCCarrillo.pdf?sequence=5

Runfola, J; Gallardo, A. (2014). Análisis comparativo de los diferentes métodos de caracterización de residuos urbanos para su recolección selectiva en comunidades urbanas. Fac. de Ciencias, Universidad de los Andes. Av. Alberto Carnevalli, Nivel Sótano, Mérida, Venezuela. Circuito Universidad de los Andes para El Manejo Integral de los Desechos (CIULAMIDE). 